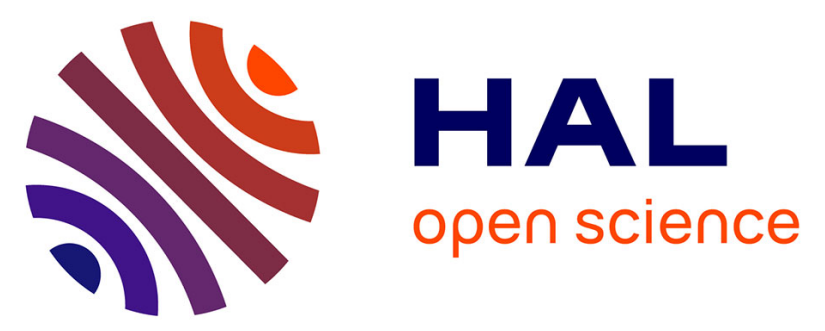

\title{
Anisotropie de susceptibilité magnétique (ASM) et chimie des traces: une nouvelle méthodologie pour démêler processus hydrothermaux et supergènes. Application au gisement à Ba-Fe-F de Chaillac (Indre, France)
}

Stanislas Sizaret, Yan Chen, Eric Marcoux, Jean-Claude Touray

\section{To cite this version:}

Stanislas Sizaret, Yan Chen, Eric Marcoux, Jean-Claude Touray. Anisotropie de susceptibilité magnétique (ASM) et chimie des traces: une nouvelle méthodologie pour démêler processus hydrothermaux et supergènes. Application au gisement à Ba-Fe-F de Chaillac (Indre, France). Comptes rendus de l'Académie des sciences. Série IIa, Sciences de la terre et des planètes, 2001, 322, pp.431-437. 10.1016/S1251-8050(01)01558-0 . hal-00089821

\section{HAL Id: hal-00089821 \\ https://hal-insu.archives-ouvertes.fr/hal-00089821}

Submitted on 5 Mar 2013

HAL is a multi-disciplinary open access archive for the deposit and dissemination of scientific research documents, whether they are published or not. The documents may come from teaching and research institutions in France or abroad, or from public or private research centers.
L'archive ouverte pluridisciplinaire HAL, est destinée au dépôt et à la diffusion de documents scientifiques de niveau recherche, publiés ou non, émanant des établissements d'enseignement et de recherche français ou étrangers, des laboratoires publics ou privés. 


\title{
Anisotropie de susceptibilité magnétique (ASM) et chimie des traces : une nouvelle méthodologie pour démêler processus hydrothermaux et supergènes. Application au gisement à Ba-Fe-F de Chaillac (Indre, France)
}

\author{
Stanislas Sizaret*, Yan Chen, Éric Marcoux, Jean-Claude Touray \\ Institut des sciences de la Terre d'Orléans (Isto), université d'Orléans, 8, rue Léonard-de-Vinci, 45072 Orléans, France
}

Reçu le 17 décembre 2000 ; accepté le 26 février 2001

Présenté par Zdenek Johan

\begin{abstract}
Anisotropy of magnetic susceptibility (AMS) and trace chemistry: a new approach to discriminate between hydrothermal and supergene processes. Application to the Ba-Fe-F deposit of Chaillac (Indre, France). Anisotropy of magnetic susceptibility (AMS) determinations and trace-element analyses have been firstly carried out, at Chaillac, on two ferruginous sandstone sections of hydrothermal and lateritic origins, respectively. The goethite has been identified as the main AMS carrier. Distinct AMS patterns and characteristic Ti-As anomalies have been observed, which allows to quantify the importance of hydrothermal and lateritic processes when both are superimposed. These preliminary results show that the 'Fe-Ba complex' of hydrothermal origin at Chaillac has been lateritized for a few meters from the present erosion surface. () 2001 Académie des sciences / Éditions scientifiques et médicales Elsevier SAS
\end{abstract}

Chaillac / ASM / hydrothermal / laterite / barytine / goethite / Massif Central / France

Résumé - L'étude a consisté à comparer l'anisotropie de susceptibilité magnétique (ASM) et les teneurs en éléments traces de grès latéritiques stériles et de grès ferrugineux hydrothermalisés riches en barytine du gisement de Chaillac. La gœthite est le principal porteur de l'ASM. Ces deux grès possèdent des ASM distinctes et des anomalies caractéristiques respectivement en Ti et As. À partir de ces résultats, il devient possible de quantifier les parts respectives de l'hydrothermalisme et de la latéritisation dans les niveaux superficiels où ces deux phénomènes sont superposés, le complexe ferro-barytique de Chaillac apparaissant ainsi comme une formation hydrothermale latéritisée dans les premiers mètres à partir de la surface actuelle. @ 2001 Académie des sciences / Éditions scientifiques et médicales Elsevier SAS

Chaillac / AMS / hydrothermal / latérite / barite / gothite / Massif central / France

\section{Abridged version}

\section{Introduction and geological setting}

Located at the southern part of the Bassin de Paris, where Triassic formations lay unconformably on the base- ment in the northwestern part of the French Massif Central, Chaillac deposits are composed of a stratiform $\mathrm{Ba}-\mathrm{Fe}$ ore body connected with a F-Ba rich vein (figure 1). The stratiform part is hosted by Hettangian fluviatile sandstones [1] with barite and goethite-rich cement, crosscut by barite and goethite banded veins. This typical facies is called 'ironbarite complex' [12].

\footnotetext{
* Correspondance et tirés à partr.

Adresse e-mail : ssizaret@esem-etud.univ-orleans.fr (S. Sizaret).
} 
Respective roles of hydrothermalism and lateritization in the genesis and evolution of this complex have been largely debated $[2,6,8,10,12]$. We propose in this study a new methodological approach to discriminate and quantify these two processes.

\section{Sampling and characterization methods}

158 oriented cores have been sampled by a gasoline drill for AMS analysis and polished sections in the $\mathrm{La}$ Raillerie and Redoutières open pits (figure 1). The cores are oriented by both magnetic and sun compasses, the difference between the two measurements being negligible.

In La Raillerie open pit the lower ferruginous level (site A) and the upper one (site B) have been sampled (figure 2). Samples from the lower part are composed of sandstones cemented by colloform goethite with hydrothermal barite. Samples from the upper part are of sandstone cemented by massive goethite.

In the Redoutières open pit, site $\mathbf{C}$ at the bottom and site D at the top of the iron-barite complex have been sampled (figure 1). For both sites, hand samples from mineralized veins and host sandstones have been collected.

CAMECA SX 50 electron microprobe has been used for analysis of goethite. AMS measurements have been carried out with a KLY3 susceptometer.

\section{Results}

\subsection{Trace-element patterns}

Two populations can be classified from the point of view of trace-element contents (table I). The first group is composed of samples from sites $\mathbf{A}$ and $\mathbf{C}$ with $\mathrm{As}_{2} \mathrm{O}_{3}$ traces, and the second one corresponds to samples from site $\mathbf{B}$, showing $\mathrm{TiO}_{2}$ traces without $\mathrm{As}_{2} \mathrm{O}_{3}$ traces.

\subsection{AMS data}

The X-ray diffraction analysis was applied to determine the mineral composition. Concerning ferromagnetic minerals, only goethite was observed, which is confirmed by chemical analyses (table I). In addition to goethite and possible barite in the collected samples, other main minerals are quartz and muscovite which have relatively low magnetic susceptibility [2,9]. Accordingly, goethite is the main carrier of magnetic susceptibility. Deformation degree $\left(P^{\prime}\right)$ shows weak deformation for both mineralised veins and country rocks (from 1.002 to 1.015; table II); however, the form parameter $(T)$ varies according to the sampling sites (table II).

From site $\mathbf{A}$ at the lower part of the sampling section in La Raillerie open pit (figure 2), AMS show a prolate dominant fabric with a negative $T$ value (table $I I$ ), the weakly inclined $\mathrm{K}_{1}$ direction is oriented to northwest and southeast, which is meanwhile parallel to the in situ vein plane.

From site $\mathbf{B}$ on the top of the sampling section in $\mathrm{La}$ Raillerie open pit (figure 2), AMS show, however, an oblate ellipsoid shape with a positive $T$ value (table II). Directions of $\mathrm{K}_{3}$ are strongly inclined and better grouped with respect to those of $\mathrm{K}_{1}$ and $\mathrm{K}_{2}$, the latter being scattered on the horizontal plane.

From site $\mathbf{C}$ on the bottom of the iron barite complex in Les Redoutières open pit (figure 3), lineation dominance is characterized for the AMS of sandstones with a negative $T$ value (table II). The well grouped and weakly inclined $\mathrm{K}_{1}$ directions are oriented to NW-SE and parallel to the plane of the mineralized vein (CG143-8SW, CG162-15SW and CG168-10SW in figure 3). Vein measurements exhibit well-clustered vertical $\mathrm{K}_{3}$ directions and $\mathrm{K}_{1}, \mathrm{~K}_{2}$ horizontal foliation, coherent with vein orientation (CF168-10SW in figure 3). We note that the SE-NW-oriented $\mathrm{K}_{1}$ direction in the vein is compatible to that observed in the host sandstones (CG168-10SW in figure 3).

From site $\mathbf{D}$ on the top of the iron barite complex in Les Redoutières (figure 4), AMS obtained from both veins and sandstone shows an oblate shape with a positive $T$ value (table $I I$ ). $\mathrm{K}_{3}$ directions are strongly inclined and well grouped; however, $\mathrm{K}_{1}$ and $\mathrm{K}_{2}$ directions are horizontally dispersed.

\section{Discussion}

In the La Raillerie open pit, $\mathrm{As}_{2} \mathrm{O}_{3}$ traces on site $\mathbf{A}$ suggest a hydrothermal origin for the lower ferruginous level with barite. AMS data show a very weak deformation $\left(P^{\prime}<2 \%\right)$; we consider the posterior tectonic influence is negligible. Textures show that goethite is generated by precipitation in the sand porosity during fluid flow, thus AMS fabric cannot be interpreted as of sedimentary (detritic) origin. Observation of the coherence between $\mathrm{K}_{1}$ direction and horizontal vein plane may indicate that this NW-SE shallow $\mathrm{K}$ direction is acquired during hydrothermal mineralisation and shows that of the hydrothermal circulation.

$\mathrm{TiO}_{2}$ traces on site $\mathbf{B}$ suggest a lateritic concentration at the upper ferruginous level [11]. This lateritized sandstone presents an oblate type of AMS comparable to that described in the iron crust from laterite. The absence of spinel and the horizontal foliation may indicate that AMS is probably acquired during laterization processes and is not an inherited fabric [5].

In Les Redoutières open pit, a similar phenomenon has been observed. On site $\mathbf{C}$, where the samples have been collected at the bottom of the iron barite complex, the prolate shape is dominant. $\mathrm{K}_{1}$ directions is better grouped with respect to the other two ones and parallel to the plane of the associated vein.

Furthermore, goethite has usually an inverse magnetic fabric [3, 7], with its cristallographic axis $c$ having the same direction as $\mathrm{K}_{3}$, and the basal section containing $\mathrm{K}_{1}$. The AMS fabric of the CF168-10SW shows that $K_{3}$ is perpendicular to the vein plane suggesting that the $c$ axis is also perpendicular to the vein. This is a common texture in hydrothermal formations. In the same way, $\mathrm{K}_{1}$ could be interpreted as a goethite alignment, or $a / b$ axis orientation along the flow direction [4]. 
However, AMS of site $\mathbf{D}$ from the top of the complex presents an oblate dominance. $\mathrm{K}_{3}$ directions are strongly inclined and well grouped; nevertheless, $\mathrm{K}_{1}$ and $\mathrm{K}_{2}$ are horizontally scattered, as observed in the lateritized sandstone on site $\mathbf{B}$, which may present a sedimentary or lateritic fabric [5].

Thus, these results show that the first metres from the top of the complex are probably lateritized. AMS results suggest a reprecipitation of iron hydroxides in the vein and sandstone. The barite is not mobilized and keeps the initial hydrothermal structure.

\section{Conclusion}

Trace chemistry and AMS studies on goethite ore provide a new tool to clarify the superposition of hydrother$\mathrm{mal} / \mathrm{sedimentary}$ and the lateritic processes. Application to Les Redoutières ore deposit shows a superficial lateritization of an hydrothermal deposit with an uncommon inherited morphology. In addition, results suggest further investigations for a possible application of AMS data to trace flow directions of ancient hydrothermal systems.

\section{Introduction et contexte géologique}

Le gisement de Chaillac, actuellement exploité pour la barytine $\left(80000 \mathrm{t} \cdot \mathrm{an}^{-1}\right)$ et la fluorine $(18000$ $\mathrm{t} \cdot \mathrm{an}^{-1}$ ), est situé au nord-ouest du Massif central, en bordure du Bassin parisien, à l'ouest de Saint-Benoîtdu-Sault (figure 1). Le cadre géologique de cette minéralisation est constitué d'un socle métamorphique paléozoïque, recouvert par des grès continentaux hettangiens datés par palynologie [1]. Le gisement présente une morphologie de gîte stratiforme connecté à un filon recoupant le socle et à remplissage de fluorine et de barytine accessoire ([10, 12], figure 1). La partie basale du gisement stratiforme est constituée d'un grès à ciment barytique (grès de base), parfois ferrugineux, actuellement exploité dans la carrière de la Raillerie. Le sommet du gisement est occupé par une chape à texture rubanée de barytine et gøthite, puissante de plusieurs mètres, recoupée par des filons subverticaux de même composition, connue sous le nom

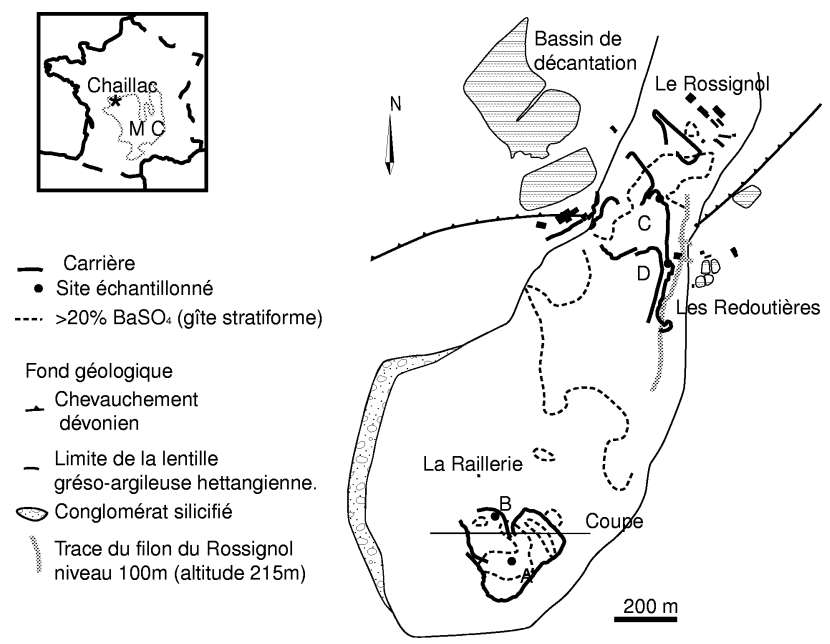

Figure 1. Carte du gisement de Chaillac. A, B, C et $\mathbf{D}$ représentent les quatre sites d'échantillonnage.

Figure 1. Map of Chaillac deposit. A, B, C and D are the four sampling zones. de complexe ferro-barytique, et interprété comme un dépôt de source thermale [12].

La genèse de ce complexe, bien représenté sur le site des Redoutières, où il recoupe et englobe des panneaux de grès ferrugineux, a donné lieu en son temps à bien des débats, notamment sur les parts respectives prises par l'hydrothermalisme et la latéritisation $[2,6,8,10,12]$.

Cette étude, réalisée sur la genèse du gisement de Chaillac, complète ce débat en proposant de quantifier, par une méthodologie originale, les ampleurs respectives de ces deux événements.

\section{2. Échantillonnage et modes opératoires}

\section{1. Échantillonnage}

Les carottes orientées utilisées pour l'ASM ont été prélevées avec une foreuse à essence et les sections polies ont été réalisées dans les carrières de la Raillerie et des Redoutières (figure 1). Les échantillons ont été orientés par les boussoles magnétique et solaire. La différence entre les deux mesures est négligeable. Dans la carrière de la Raillerie, les grès du niveau ferrugineux inférieur (site $\mathbf{A}$ ) et du niveau ferrugineux supérieur (site B), séparés par un intercalaire stérile pauvre en $\mathrm{Fe}$ et $\mathrm{Ba}$, ont été échantillonnés (figure 2). Dans le site $\mathbf{A}$, le ciment du grès est composé de gœthite à texture collomorphe rubanée et de barytine hydrothermale en quantités notables. Dans le site $\mathbf{B}$, il s'agit de grès quartzeux, avec de la gothite en grains cimentés par de la gothite massive.

Deux sites ont été échantillonnés dans le complexe ferro-barytique de la carrière des Redoutières : le site C (base du complexe, $5 \mathrm{~m}$ sous la surface actuelle), et le site $\mathbf{D}$ (sommet du complexe, $1,5 \mathrm{~m}$ sous la surface actuelle). De bas en haut, il n'y a qu'un niveau ferrugineux, sans intercalaire stérile. Les filons et leur encaissant ont été échantillonnés à la base de ce niveau (site C) et au sommet (site D) (figure 1). Microscopiquement, les échantillons ont une texture 


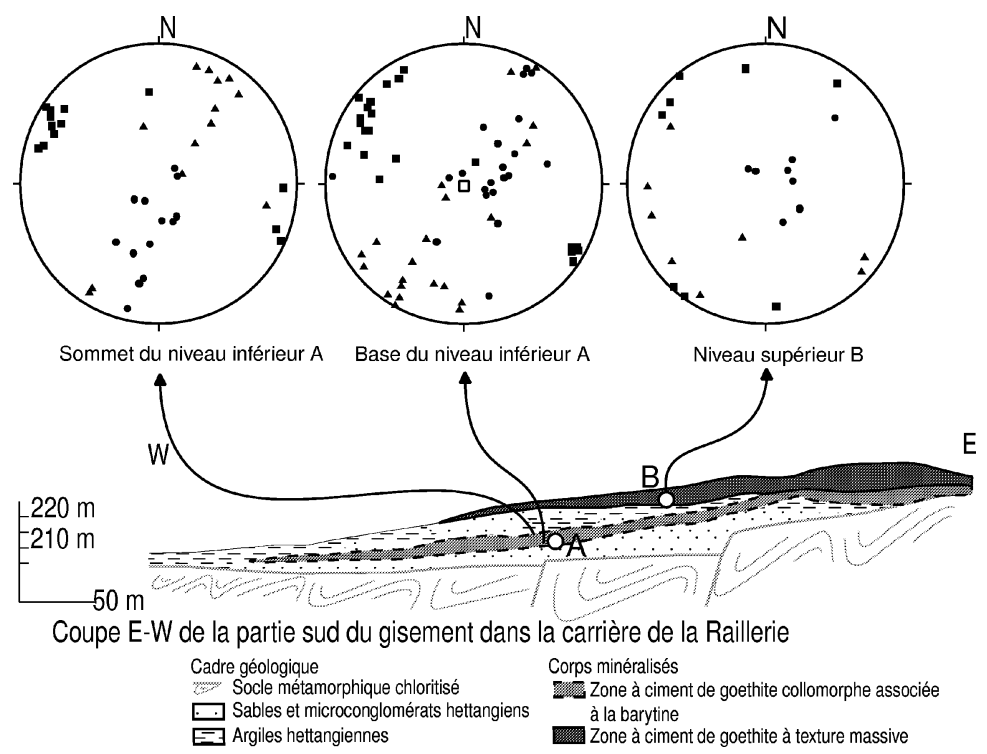

Figure 2. Stéréogrammes de l'ASM pour les sites A et B, avec leurs positions sur la coupe géologique (figure 1). Seul un filon barytique horizontal a été observé à la base du niveau inférieur de la Raillerie. Sur les stéréogrammes, les carrés pleins, triangles, cercles et carré vides représentent respectivement $K_{1}, K_{2}, K_{3}$ et pôle du filon.

Figure 2. ASM stereographic projection for sites $\mathbf{A}$ and B with their sampling positions in the geologic section (figure 1). A unique horizontal baritic vein has been found at the basis of inferior level of "La Raillerie". On the stereographic projection solid squares, triangles, circles and open square refer to $K_{1}, K_{2}, K_{3}$ and vein pole, respectively. rubanée à alternance de lits de barytine et de goethite collomorphe.

\subsection{Modes opératoires}

Les sections polies ont été analysées à la microsonde électronique Cameca SX 50. L'anisotropie de susceptibilité magnétique (ASM) des carottes a été mesurée à l'aide du susceptomètre Kappa-bridge KLY3 au laboratoire de magnétisme de roches d'Orléans. Les mesures ont porté sur 34 échantillons (site A), 8 échantillons (site B), 98 échantillons (site C) et 18 échantillons (site D). Pour chaque échantillon, la susceptibilité magnétique a été mesurée dans trois plans de l'espace, afin de construire les ellipsoïdes d'anisotropie.

\section{Résultats}

\subsection{Compositions chimiques des gothites des ciments des grès}

115 analyses ont été réalisées. Deux groupes d'échantillons se distinguent selon les sites d'échantillonnage (tableau I). Un premier ensemble, regroupant les échantillons des sites A (La Raillerie, niveau inférieur) et $\mathbf{C}$ (les Redoutières, niveau inférieur), se caractérise par une anomalie positive en $\mathrm{As}_{2} \mathrm{O}_{3}$, de l'ordre de $0,15 \%$.

Le deuxième ensemble caractérise les échantillons du site B (La Raillerie, niveau supérieur), avec une anomalie positive en $\mathrm{TiO}_{2}$, une très faible anomalie en $\mathrm{As}_{2} \mathrm{O}_{3}$ et une teneur plus basse en $\mathrm{Al}_{2} \mathrm{O}_{3}$. Ces résultats sont propres au ciment de ce niveau.

La gothite en grains du site $\mathbf{B}$ ne montre pas de teneurs significatives en $\mathrm{TiO}_{2}$ et $\mathrm{As}_{2} \mathrm{O}_{3}$.

\subsection{Anisotropie de susceptibilité magnétique (ASM)}

Des diagrammes de diffraction $\mathrm{X}$ ont permis de déterminer la composition minéralogique des échantillons. Concernant les minéraux magnétiques, la gœthite est identifiée comme le minéral principal. Les observations microscopiques indiquent que, à part la gothite, les principaux minéraux constitutifs sont le quartz et la muscovite; ces derniers ont une susceptibilité magnétique nettement inférieure à celle de la gothite, qui est donc le porteur principal de l'ASM dans les échantillons de cette étude [3,9]. Une faible valeur de degré de déformation a été observée dans l'ensemble de la collection entre 1,002 et 1,015 (tableau II) ; cependant, le paramètre de forme $(T)$ varie selon les sites de prélèvement (tableau II).

\section{Dans la carrière de la Raillerie}

- Niveau ferrugineux inférieur (site A ; figure 2)

L'ASM du grès du site $\mathbf{A}$ est caractérisée par un ellipsoïde, allongé avec un paramètre de forme négatif $T \approx-0,3$ (tableau II). La direction SE-NW de l'allongement $\left(\mathrm{K}_{1}\right)$ est portée par le plan du filon le plus proche. Les trois directions de l'ellipsoïde de susceptibilité magnétique sont relativement groupées.

- Niveau ferrugineux supérieur (site B ; figure 2)

L'ASM du grès du site $\mathbf{B}$ a un ellipsoïde aplati avec un paramètre de forme positif $(T=0,05)$ (tableau II). La direction de $\mathrm{K}_{3}$ est peu dispersée, les directions de $\mathrm{K}_{1}$ et $\mathrm{K}_{2}$ sont, cependant, dispersées horizontalement, marquant le plan de foliation de la susceptibilité magnétique.

\section{Dans la carrière des Redoutières}

- Base du niveau ferrugineux (site C ; figure 3)

L'ASM du grès du site $\mathbf{C}$ se distingue par une anisotropie peu allongée et un paramètre de forme négatif 
Tableau I. Résultats d'analyses à la microsonde électronique des différents faciès étudiés. Les résultats sont donnés à $3 \sigma$ (intervalle de confiance à $99 \%$ ). Pour les éléments traces, deux moyennes encadrant la moyenne vraie ont étés calculées : une moyenne inférieure où les valeurs égales ou inférieures au seuil de détection sont considérées égales à $0(X>)$, une moyenne supérieure où les valeurs égales ou inférieures au seuil de détection sont considérées égales à ce seuil ( $>X)$. Pour chaque élément, le standard est donné entre parenthèses : $\mathrm{As}(\mathrm{AsGa}), \mathrm{Fe}\left(\mathrm{Fe} \mathrm{O}_{3}\right), \mathrm{Al}\left(\mathrm{Al} \mathrm{O}_{3}\right), \mathrm{Ti}_{2}$ et $\mathrm{Mn}\left(\mathrm{MnTiO}_{2}\right), \mathrm{P}\left(\mathrm{Ca}_{5}\left(\mathrm{PO}_{4}\right)_{3}(\mathrm{OH})_{2}\right)$. Mesures réalisées sous une tension d'accélération de $15 \mathrm{kV}$, une intensité de $12 \mathrm{nA}$ et des temps de comptage de $20 \mathrm{~s}$ pour tous les éléments.

Table I. Results of electronic microprobe analysis. Results are given at $3 \sigma(99 \%$ confidence). For traces elements two averages framing the true value have been calculated: a lower average where the values which are below the detection limit are considered equal to $0(X>)$, and an upper average where the values which are below the detection limit are considered as equal to this limit. For each element standard is given between brackets: $\mathrm{As}(\mathrm{AsGa}), \mathrm{Fe}\left(\mathrm{Fe}_{2} \mathrm{O}_{3}\right), \mathrm{Al}\left(\mathrm{Al}_{2} \mathrm{O}_{3}\right)$, Ti and $\mathrm{Mn}\left(\mathrm{MnTiO}_{2}\right), \mathrm{P}\left(\mathrm{Ca}_{5}\left(\mathrm{PO}_{4}\right)_{3}(\mathrm{OH})_{2}\right)$. Analyses have been performed with a tension of $15 \mathrm{kV}$, an intensity of $12 \mathrm{nA}$ and a counting time of $20 \mathrm{~s}$.

\begin{tabular}{|c|c|c|c|c|c|c|c|c|c|c|c|c|c|c|c|}
\hline & $\% \mathrm{pds}$ & $\mathrm{Fe}_{2} \mathrm{O}_{3}$ & $\mathrm{Al}_{2} \mathrm{O}_{3}$ & $\mathrm{SiO}_{3}$ & $\mathrm{P}_{2} \mathrm{O}_{5}>$ & $>\mathrm{P}_{2} \mathrm{O}_{5}$ & $\mathrm{MnO}>$ & $>\mathrm{MnO}$ & $\mathrm{As}_{2} \mathrm{O}_{3}>$ & $>\mathrm{As}_{2} \mathrm{O}_{3}$ & $\mathrm{TiO}_{2}>$ & $>\mathrm{TiO}_{2}$ & $\mathrm{H}_{2} \mathrm{O}>$ & $>\mathrm{H}_{2} \mathrm{O}$ & Total \\
\hline \multirow{2}{*}{$\begin{array}{l}\text { Raillerie } \mathbf{A} \text {, grès ferrugineux } \\
\text { du niveau inférieur }\end{array}$} & Moyenne & 79,07 & 1,45 & 3,97 & 0,15 & 0,16 & 0,03 & 0,08 & 0,12 & 0,15 & 0,01 & 0,05 & 15,07 & 15,20 & 100,00 \\
\hline & $\sigma$ & 1,10 & 0,55 & 0,66 & 0,11 & 0,09 & 0,05 & 0,03 & 0,10 & 0,07 & 0,02 & 0,01 & 0,78 & 0,77 & \\
\hline \multirow{2}{*}{$\begin{array}{l}\text { Raillerie B, ciment de gœthite } \\
\text { du niveau ferrugineux supérieur }\end{array}$} & Moyenne & 82,53 & 0,98 & 1,61 & 0,04 & 0,07 & 0,01 & 0,07 & 0,01 & $\mathbf{0 , 0 7}$ & 0,11 & 0,12 & 14,57 & 14,73 & 100,00 \\
\hline & $\sigma$ & 1,56 & 0,44 & 1,10 & 0,05 & 0,03 & 0,02 & 0,00 & 0,02 & 0,00 & 0,07 & 0,05 & 1,25 & 1,26 & \\
\hline \multirow{2}{*}{$\begin{array}{l}\text { Raillerie } \mathbf{B} \text {, grain de gœthite } \\
\text { du niveau supérieur }\end{array}$} & Moyenne & 84,24 & 0,37 & 1,48 & 0,05 & 0,06 & 0,00 & 0,07 & $\mathbf{0 , 0 0}$ & $\mathbf{0 , 0 7}$ & 0,02 & 0,05 & 13,65 & 13,83 & 100,00 \\
\hline & $\sigma$ & 0,62 & 0,18 & 0,18 & 0,03 & 0,02 & 0,00 & 0,00 & 0,00 & 0,00 & 0,03 & 0,01 & 0,67 & 0,67 & \\
\hline \multirow[t]{2}{*}{ Redoutières $\mathbf{C}$, gœethite des filons } & Moyenne & 84,80 & 1,17 & 2,70 & 0,03 & 0,06 & 0,03 & 0,08 & 0,20 & 0,21 & $\mathbf{0 , 0 0}$ & 0,05 & 10,93 & 11,08 & 100,00 \\
\hline & $\sigma$ & 5,30 & 0,57 & 0,86 & 0,05 & 0,03 & 0,06 & 0,03 & 0,13 & 0,12 & 0,02 & 0,01 & 5,23 & 5,23 & \\
\hline
\end{tabular}

Tableau II. Résultats statistiques des paramètres de l'ASM [6]. $K v:$ susceptibilité volumique, $L$ et $F:$ linéation et foliation de susceptibilité magnétique, $P^{\prime}:$ degré normalisé d'anisotropie, $T$ : forme de l'anisotropie. $\mathrm{K}_{1}, \mathrm{~K}_{2}$ et $\mathrm{K}_{3}$ : directions principales de l'ASM, $D$ et $I$ : direction et inclinaison, $\alpha_{x} 95$ et $\alpha_{y 95}$ : intervalle de confiance statistique à $95 \%$.

Table II. Statistic AMS parameters [6]. $K v$ : bulk susceptibility, $L$ and $F$ : lineation and foliation, $P^{\prime}$ : normalised anisotropy, $T$ : the anisotropy shape. $\mathrm{K}_{1}, \mathrm{~K}_{2}, \mathrm{~K}_{3}$ : principal ellipsoid directions, $D$ and $I$ : declination and inclination, $\alpha_{x} 95$ and $\alpha_{y 95}$ : the $95 \%$ statistic intervals of confidence.

\begin{tabular}{|c|c|c|c|c|c|c|c|c|c|c|c|c|c|c|c|c|c|c|}
\hline \multirow[b]{2}{*}{ Site } & \multirow[b]{2}{*}{$\mathrm{nb}$} & \multirow[b]{2}{*}{$K v\left(10^{-5} \mathrm{SI}\right)$} & \multirow[b]{2}{*}{$L$} & \multirow[b]{2}{*}{$F$} & \multirow[b]{2}{*}{$P^{\prime}$} & \multirow[b]{2}{*}{$T$} & \multicolumn{4}{|c|}{$\mathrm{K}_{1}$} & \multicolumn{4}{|c|}{$\mathrm{K}_{2}$} & \multicolumn{4}{|c|}{$\mathrm{K}_{3}$} \\
\hline & & & & & & & $D\left({ }^{\circ}\right)$ & $I\left({ }^{\circ}\right)$ & $\alpha_{x 95}$ & $\alpha_{y 95}$ & $D\left({ }^{\circ}\right)$ & $I\left({ }^{\circ}\right)$ & $\alpha_{x 95}$ & $\alpha_{y 95}$ & $D\left(^{\circ}\right)$ & $I\left({ }^{\circ}\right)$ & $\alpha_{x 95}$ & $\alpha_{y 95}$ \\
\hline $\mathbf{A}$ : grès inférieur de la Raillerie Bas & 13 & 36 & 1,010 & 1,005 & 1,015 & $-0,312$ & 303,9 & 12,5 & 6,6 & 10,2 & 211,1 & 17,3 & 8,3 & 15,7 & 66,1 & 65,9 & 9,5 & 16,2 \\
\hline $\mathbf{A}$ : grès inférieur de la Raillerie Haut & 21 & 28 & 1,005 & 1,002 & 1,007 & $-0,347$ & 297,5 & 9,0 & 4,6 & 12,2 & 34,1 & 24,5 & 11,8 & 17,6 & 189,3 & 61,3 & 5,9 & 17,1 \\
\hline B : grès supérieur de la Raillerie & 8 & 48 & 1,001 & 1,002 & 1,003 & 0,059 & 353,8 & 6,1 & 8,8 & 32,4 & 265,9 & 15,9 & 13,9 & 32,5 & 72,3 & 77,5 & 11,0 & 15,9 \\
\hline $\mathrm{C}:$ grès $162-15 \mathrm{SW}$ & 29 & 47 & 1,003 & 1,002 & 1,005 & $-0,088$ & 291,6 & 7 & 7,7 & 11,4 & 90,8 & 81,5 & 11,1 & 14,1 & 19,7 & 4 & 8,6 & 15,3 \\
\hline C : grès143-8SW & 13 & 57 & 1,001 & 1,001 & 1,003 & 0,001 & 116,3 & 1,1 & 13,6 & 17,9 & 346,8 & 84,9 & 15,5 & 22,2 & 31,5 & 0,7 & 15,7 & 20,4 \\
\hline C : grès 168-10SW & 12 & 33 & 1,001 & 1,001 & 1,002 & $-0,230$ & 323,9 & 2,7 & 10,2 & 18,7 & 70,5 & 73,1 & 15,7 & 28,1 & 233,7 & 16,4 & 17,1 & 31,0 \\
\hline C : filon 168-10SW & 30 & 50 & 1,002 & 1,003 & 1,005 & 0,137 & 297,7 & 4,5 & 4,0 & 11,7 & 38,8 & 16,2 & 11,1 & 14,8 & 196,4 & 76,2 & 4,2 & 14,2 \\
\hline D : grès 108-28NE & 4 & 28 & 1,002 & 1,012 & 1,015 & 0,599 & 107,8 & 8,4 & 9,1 & 18,3 & 20,1 & 5,8 & 8,5 & 18,1 & 353,4 & 80,1 & 3,6 & 12,1 \\
\hline D : filon 108-28NE & 14 & 40 & 1,001 & 1,010 & 1,012 & 0,808 & 211,4 & 5,7 & 8,6 & 39,6 & 122 & 10 & 6 & 39,4 & 256,5 & 83,4 & 6,1 & 11,3 \\
\hline
\end{tabular}


S. Sizaret et al. / C. R. Acad. Sci. Paris, Sciences de la Terre et des planètes / Earth and Planetary Sciences 332 (2001) 431-437
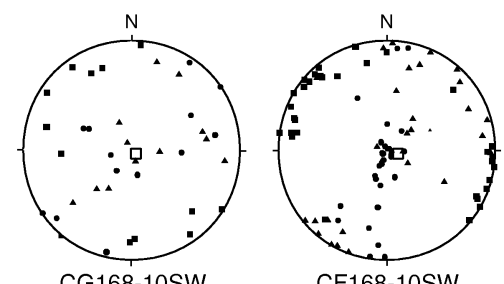

CG168-10SW

CF168-10SW
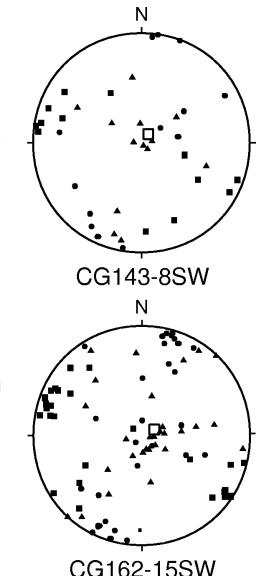

CG162-15SW

Figure 3. Stéréogrammes représentant les directions de l'ASM sur le site $\mathbf{C}$ ( $\mathbf{G}$ pour grès et $\mathbf{F}$ pour filon). Les stéréogrammes regroupent les échantillons de même nature lithologique, situés dans le voisinage d'un même filon. Le bloc diagramme schématise la zone d'échantillonnage. Les filons échantillonnés sont représentés avec leurs épaisseurs. Les filons qui n'ont pas pu être échantillonnés sont représentés par un trait. La surface topographique actuelle est à l'altitude $209 \mathrm{~m}$. Symboles : voir la figure 2.

Figure 3. Stereonet of the AMS directions for the site $\mathbf{C}$ ( $\mathbf{G}$ for sandstone and $\mathbf{F}$ for vein). The stereo associate samples according to their lithology and their position close to a same vein. The diagram schematises the sampled site. For sampled veins, thickness is given. Veins that have not been sampled are figured by a line. The current topographic surface is at $209 \mathrm{~m}$. Symbol: see figure 2 .

ou proche de $0(T<0,002)$ (tableau II). La direction de $\mathrm{K}_{1}$ est portée par le plan du filon le plus proche. Un seul filon a été échantillonné (CF168-10SW) et son anisotropie est aplatie $(T=0,137)$. Le plan marqué par $\mathrm{K}_{1}$ et $\mathrm{K}_{2}$ est parallèle au plan du filon, la direction de $\mathrm{K}_{3}$ est bien groupée et perpendiculaire au plan du filon. Ce plan porte une linéation magnétique SENW parallèle à l'allongement $\left(\mathrm{K}_{1}\right)$ mesuré dans l'encaissant gréseux immédiat (CG168-10SW) (figure 3). Les allongements de l'ASM des grès CG143-8SW et CG162-15SW sont eux aussi parallèles à cette linéatation magnétique SE-NW.

- Sommet du niveau ferrugineux (site $\mathbf{D}$; figure 4) Les directions de $\mathrm{K}_{1}$ et de $\mathrm{K}_{2}$ sont dispersées horizontalement et les directions de $\mathrm{K}_{3}$ sont groupées verticalement. Le filon et le grès ont la même anisotropie aplatie suivant le plan horizontal $(T>0,5)$ ( $t a-$ bleau II).

\section{Discussion}

La composition chimique du ciment du grès inférieur de la Raillerie (site A), notamment l'anomalie en arsenic, confirme une origine pro parte hydrothermale.

Le paramètre de degré de déformation est généralement faible $\left(P^{\prime}<2 \%\right)$, ce qui indique que la tectonique régionale, de direction $\mathrm{N} 20$, n'a probablement

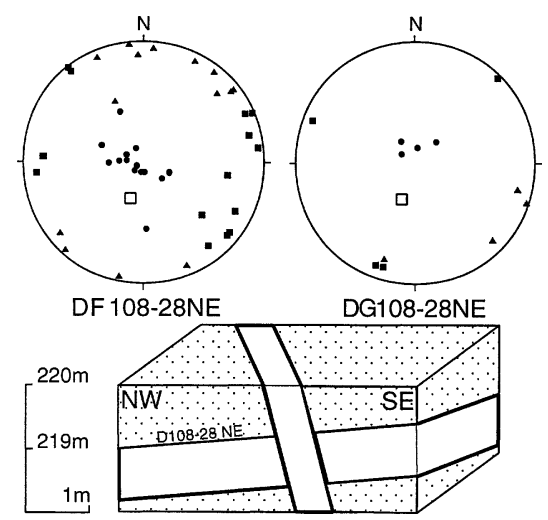

Figure 4. Stéréogrammes représentant les directions de l'ASM sur le site D. La surface topographique actuelle est à l'altitude $220 \mathrm{~m}$ (voir la figure 2 pour la légende complète).

Figure 4. Stereographic projection of the AMS directions on site D. Current topographic surface is at $220 \mathrm{~m}$ (for complete caption, see figure 2).

pas affecté la zone étudiée. De plus, l'observation des textures montre que la gœthite porteuse de l'ASM s'est formée par précipitation chimique dans la porosité d'un grès, à partir d'un fluide en mouvement. Les fabriques d'ASM ne peuvent donc pas être interprétées comme des fabriques sédimentaires détritiques.

Enfin, ce grès minéralisé est caractérisé par une fabrique d'ASM allongée horizontalement suivant $K_{1}$. Cette direction SE-NW est portée par le plan du filon le plus proche et suggère la direction possible de circulation hydrothermale dans ce grès.

Le grès du niveau supérieur de la Raillerie (site $\mathbf{B}$ ) présente une anomalie en $\mathrm{TiO}_{2}$, composé peu mobile se concentrant dans les latérites [11]. Il passe en profondeur à un sable silteux avec de la gothite détritique en grains. Les grains de gothite observés dans les échantillons du site $\mathbf{B}$, l'absence d'anomalie en arsenic et l'anomalie en $\mathrm{TiO}_{2}$ suggèrent que ce niveau ferrugineux est une latérite développée sur un sable à particules de gothite. Ce grès latéritisé se distingue du grès hydrothermalisé du site $\mathbf{A}$ par un ellipsoïde d'ASM aplati, avec une direction de $\mathrm{K}_{3}$ verticale. Une récente étude sur les ASM des latérites montre qu'en l'absence de spinelles la fabrique de l'ASM est héritée dans les horizons profonds et isotrope, avec une légère compaction au niveau de la croûte ferrugineuse [5]. L'ASM du site B est donc, soit héritée du sable sous-jacent, soit d'origine latéritique. La texture observée au microscope montre peu de grains préservés avec un ciment gothitique massif. Ces observations nous conduisent à privilégier l'hypothèse d'une ASM latéritique, avec une influence très faible de l'héritage sédimentaire.

Les ASM définies sur les sites de la Raillerie nous ont permis d'interpréter les ASM du complexe ferrobarytique des Redoutières. 
La forme de l'ellipsoïde d'ASM du site $\mathbf{C}$ (base du complexe) est allongée, la direction de $\mathrm{K}_{1}$ est groupée et portée par le plan des filons associés. Cette ASM est comparable à celle du site $\mathbf{A}$.

Échantillonné à $5 \mathrm{~m}$ sous la surface actuelle, à la base du niveau ferrugineux des Redoutières, ce niveau est très peu affecté par la latéritisation. De plus, dans le cas du filon (CF168-10SW), nous pouvons mener l'interprétation plus loin. La gœthite est connue pour présenter une fabrique inverse, c'est-à-dire que $\mathrm{K}_{3}$ de l'ASM et l'axe cristallographique $c$ de la gothite sont parallèles [3, 7]. L'ASM de ce filon montre $\mathrm{K}_{3}$ verticalement groupé, suggérant une orientation de l'axe $c$ des gœthites perpendiculairement au plan du filon. De telles textures sont courantes dans les formations hydrothermales. De même, l'orientation de $\mathrm{K}_{1}$ pourrait être due aux alignements des cristaux de gothite ou à une orientation préférentielle des axes $a$ et $b$. Cette texture serait la conséquence de courants hydrothermaux [4].

L'ellipsoïde d'ASM du site D (sommet du complexe ferro-barytique) est aplati, la direction de $\mathrm{K}_{3}$ est verticale et les directions de $\mathrm{K}_{1}, \mathrm{~K}_{2}$ sont dispersées dans le plan horizontal. Cette ASM est semblable à celle de la latérite du site $\mathbf{B}$. Les échantillons ont été prélevés à $1,5 \mathrm{~m}$ sous la surface topographique actuelle. Ces résultats confirment que le sommet du niveau ferro-barytique des Redoutières peut être qualifié de latérite. Un âge sidérolithique du phénomène de latéritisation est probable, dans la mesure où il est bien représenté régionalement [2].
Nos observations suggèrent que la latéralisation n'affecte que les premiers mètres du complexe ferrobarytique des Redoutières. Les résultats de l'ASM suggèrent, en effet, que les hydroxydes de fer ont été reprécipités in situ dans les grès et les filons. La barytine étant peu soluble dans les conditions de surface, la phase de latéritisation n'a donc pas perturbé la structuration filonienne, encore bien visible dans ce complexe. Cette structuration est donc une morphologie filonienne héritée, et non une structuration d'origine latéritique.

\section{Conclusions}

Menées conjointement sur des minerais à gœthite, la chimie des traces et l'anisotropie de susceptibilité magnétique sont deux méthodes dont l'association forme un outil nouveau pour reconnaître des superpositions de processus latéritique et hydrothermal.

Les résultats de cette étude confirment l'existence d'une latéritisation superficielle du dépôt hydrothermal de Chaillac, donnant lieu à une morphologie latéritique peu commune. Cette méthodologie peut s'étendre à d'autres sites minéralisés pour aider à résoudre des problèmes génétiques comparables.

Enfin, au-delà d'une utilisation empirique, ces résultats suggèrent la possibilité d'utiliser les données ASM pour un traçage des directions des écoulements hydrothermaux fossiles.

Remerciements. Nous remercions F. Pinier, directeur de Barytine de Chaillac, et J.-P. Chambard, directeur de la Société industrielle du Centre, qui, par leurs soutiens, ont concouru à la réalisation de ce travail et autorisé la publication des résultats. Nous adressons nos remerciements à P. Rochette pour ses commentaires constructifs, qui nous ont permis de mener plus loin nos réflexions.

\section{Références}

[1] Châteauneuf J.-J., Étude de la microflore dans le gisement barytique de Chaillac, Mém. BRGM 104 (1980) 375-376.

[2] Deurbergue A., Transformation post-sédimentaire à l'interface Paléogène-Mézosoïque sur la bordure nord du Massif central. Altération, diagénèse, concentrations minérales, thèse de $3^{\mathrm{e}}$ cycle, université d'Orléans, 1984.

[3] Dunlop D.J., Özdemir Ö., Iron oxyhydroxides, in: Rock Magnetism Fundamentals and Frontiers, Cambridge University Press, 1997, pp. 74-76.

[4] Kesler S.E., Stoiber R.E., Billings G.K., Direction of flow mineralizing solutions at Pine Point, N.W.T., Econ. Geol. 67 (1972) 19-24.

[5] Mathé P.E., Rochette P., Vandamme D., Colin F., Volumetric changes in weathered profiles: iso-element mass balance method questioned by magnetic fabric, Earth Planet. Sci. Lett. 167 (1999) 255-267.

[6] Parron C., Nahon D., Tardy Y., Cuirassements ferrugineux et barytiques par altération latéritique des grès hettangiens dans la région de Chaillac (Indre), Mém. BRGM 104 (1980) 385-396.
[7] Rochette P., Jackson M., Aubourg C., Rock magnetism and the interpretation of anisotropy of magnetic susceptibility, Rev. Geophys. 30 (3) (1992) 209-226.

[8] Tardy Y., Clauer N., Fontes J.-C., Nahon D., Origine marine des éléments constituant les barytines de la région de Chaillac (Indre, France). Relation probable entre transgressions et circulations convectives d'eau de mer dans les massifs granitiques, C. R. Acad. Sci. Paris, série II 295 (1982) 219-224.

[9] Tarling D.H., Hrouda F., The magnetic anisotropy of rocks, Chapman \& Hall, London, 1993.

[10] Touray J.C., Ziserman A., Sur la genèse des gisements (FBa-Fe-Mn) de Chaillac, Mém. BRGM 104 (1980) 377-380.

[11] Wilhelm E., Kosakévitch A., Utilisation des chapeaux de fer comme guide de prospection, Bull. BRGM II (2/3) (1979) 109-140.

[12] Ziserman A., Les gisements de Chaillac (Indre) : la barytine des Redoutières, la fluorine du Rossignol. Association d'un gîte stratiforme de couverture et d'un gîte filonien du socle, Mém. BRGM 104 (1980) 344-374. 